\title{
Highly Circularly Polarized Electroluminescence from a Chiral Europium Complex
}

\author{
Francesco Zinna; Umberto Giovanella; Lorenzo Di Bari* \\ F. Zinna; Prof. L. Di Bari \\ Dipartimento di Chimica e Chimica Industriale, Università di Pisa, via Moruzzi 3, I-56126 Pisa, Italy \\ Dr. U. Giovanella \\ Istituto per lo Studio delle Macromolecole (ISMAC), CNR Via Bassini 15, 20133 Milano, Italy \\ E-mail: lorenzo.dibari@unipi.it
}

Keywords: (OLED, circularly polarized luminescence, lanthanides, europium, chiral complexes)

Organic light-emitting diodes (OLEDs) represent an attractive technology to fabricate efficient energy-saving light sources with high color quality, wide viewing angles and a true black color. ${ }^{[1]}$

Although OLEDs available on the market, in the form of OLED lamps, OLED-TVs, OLED displayequipped portable devices, generate exclusively unpolarized electroluminescence (EL) emission, investigation on linearly polarized EL emission of organic systems ${ }^{[2]}$ has been stimulated in the last years by development of stereoscopic 3D-displays. Circularly polarized (CP) light has further found intriguing photonic applications such as molecular photoswitches, ${ }^{[3]}$ optical data storage, ${ }^{[4]}$ optical quantum information $^{[5]}$ and spintronics ${ }^{[6]}$ and its use in other fields, such as chirality sensing ${ }^{[7]}$ or enhanced image contrast in advanced medical imaging techniques, ${ }^{[8]}$ appears promising.

The ability to control the polarization of the emitted light in conjugated molecular and polymeric systems is a fascinating issue in the design of new materials. While this is well established for CP photoluminescence (PL), the direct generation of CP electroluminescence (EL) still lags behind. Yet, it would be more efficient in terms of device simplicity (thickness), production costs and integration with other organic optoelectronic devices (transistors, photodetectors), with respect to conventional CP devices employing polarizing filters. ${ }^{[9]}$

In order to achieve CPEL, the emitting material must be chiral and non racemic, in addition to all the expected features for electroluminescent devices.

CP-OLED performance can be measured by the EL dissymmetry factor $g_{\mathrm{EL}} \quad\left(\left|g_{\mathrm{EL}}\right| \leq 2\right.$, $g_{\mathrm{EL}}=2\left(\mathrm{I}_{\mathrm{L}}-\mathrm{I}_{\mathrm{R}}\right) /\left(\mathrm{I}_{\mathrm{L}}+\mathrm{I}_{\mathrm{R}}\right), \mathrm{I}_{\mathrm{L}}$, and $\mathrm{I}_{\mathrm{R}}$, being the left- and right polarized intensity, respectively). ${ }^{[10]}$ 


\section{WILEY-VCH}

Direct CPEL generation was first demonstrated in a chiral-substituted poly(p-phenylenevinylene) (PPV) derivative ${ }^{[11]}$ and since then two main strategies have been followed in the design of such devices: decorating the light-emitting polymer with chiral sidechains, or doping it with a chiral molecule (Figure 1). Geng et al. ${ }^{[12]}$ achieved the highest $g_{E L}$ value of 0.35 reported so far, by employing a hyperbranched poly(fluorenevinylene). Oda et al. ${ }^{[9]}$ measured $g_{\mathrm{EL}}$ of 0.16 and -0.25 for liquid-crystal chiral-substituted polyfluorene-based OLED. In these cases, circular polarization stems from the chiral supramolecular organization of the active layer, which may be strongly temperature- and possibly batch-dependent. ${ }^{[13]}$

Following the second approach, Fuchter et al. ${ }^{[14]}$ obtained a $g_{\text {EL }}$ value of 0.2 by doping a conventional (achiral) polymer with a helicene as the chiral inducer. An advantage of this strategy consists in the use of a previously optimized and efficient electroluminescent material, although the considerable amounts of the chiral inducer can perturb the emitter properties and affect the homogeneity of the active layer.

As typically occurs for polymer-based OLEDs, the emission band is broad, covering a relevant part of the visible spectrum; interestingly, the $g_{\mathrm{EL}}$ value remains almost constant throughout the band.

Although wide bands and constant $g$ values may be desirable for certain applications, ${ }^{[4-6]}$ there are other ones for which nearly monochromatic emission is requested. ${ }^{[15,16]}$ Furthermore, higher levels of CPEL are essential in order to switch to practical application in chiral optoelectronics and photonics.

This can be attained in OLEDs based on lanthanide complexes; such OLEDs feature very sharp emissions, whose energies are only slightly affected by the temperature and the hosting medium. ${ }^{[17]}$ Moreover, Eu(III)-based OLEDs have potential for a high electroluminescence internal quantum efficiency, thanks to their ability of harvesting triplets for light emission. Indeed, Eu(III)-based OLEDs are now an established technology for standard non-polarized electroluminescent devices $^{[15,18]}$ and recently devices embedding $\mathrm{Eu}(\mathrm{III})$ beta-diketonate complexes with external quantum efficiency (EQE) exceeding $4 \%{ }^{[19]}$ and tunable color emission have been reported. ${ }^{[20]}$

Another intriguing feature of $\mathrm{Ln}(\mathrm{III})$ complexes is that some chiral compounds display a highly CP PL, ${ }^{[10]}$ but notably their use as circularly polarized electroluminescent emitters has never been investigated so far. 


\section{WILEY-VCH}

a)

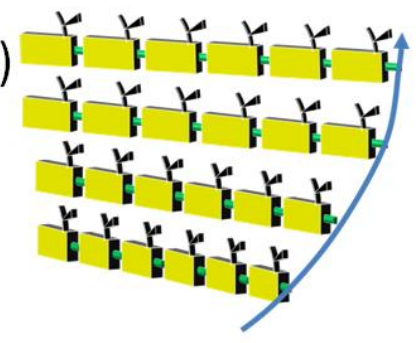

b)

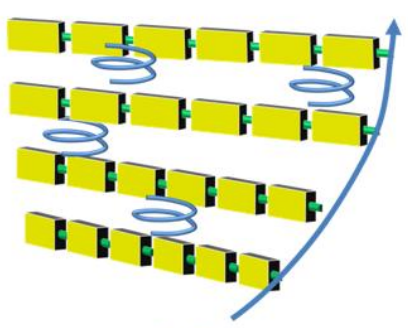

c)

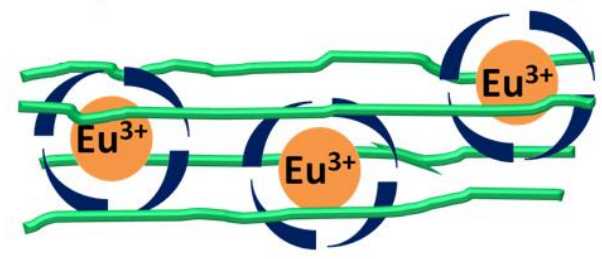

\section{Figure 1}

Three strategies employed in CP-OLED: a) polymers with chiral sidechains, able to form chiral supramolecular aggregates; b) a chiral small molecule dopant, inducing chirality on an achiral emissive polymer; c) our approach based on emissive small chiral molecules, endowed with high intrinsic polarized emission dispersed in a polymer host. This figure has only a diagrammatic purpose.

In this paper we present a completely novel approach for manufacturing an OLED with high intrinsic circularly polarized sharp red emission with a level of CPEL as high as $70 \%$, largely outperforming previously reported results (Figure 1). For the first time, we used a chiral lanthanide complex as the guest emitter in a well established solution-processed polymer-based device and we investigated the critical factors affecting $g_{\mathrm{EL}}$ value in CP-OLED.

For this prototype device, the $\mathrm{Ln}(\mathrm{III})$ complex $\mathrm{CsEu}(\mathrm{hfbc})_{4}$ (hfbc $=3$-heptafluorobutylyrylcamphorate; Figure 2a), with the highest available CP PL ( $g_{\mathrm{PL}}=1.38$ at $595 \mathrm{~nm}$, meaning $a I_{L} / I_{R}$ ratio of 85:15) for a lanthanide system, ${ }^{[21]}$ was selected as the chiral emitter. Even though it has a low PL quantum yield (3\% as powder), for our purposes, it provides a very good trade-off between polarization and total luminescence. 
WILEY-VCH

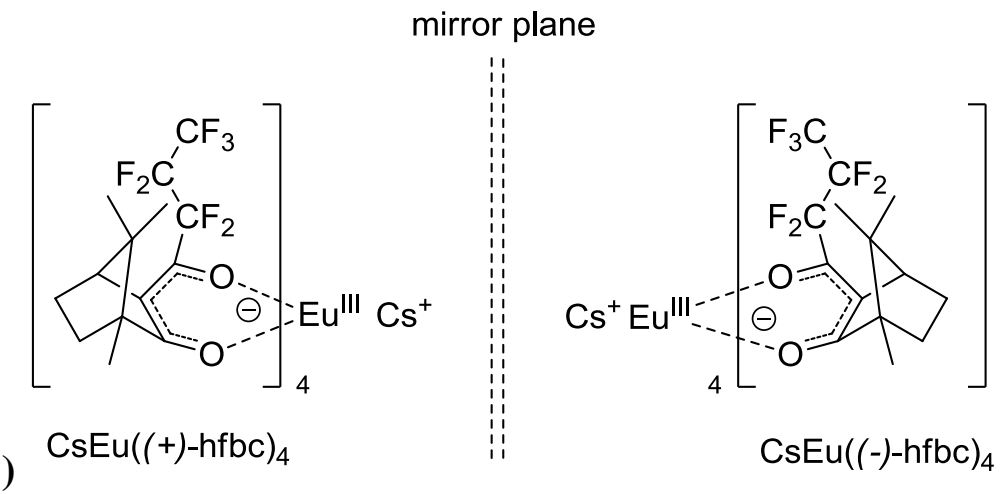

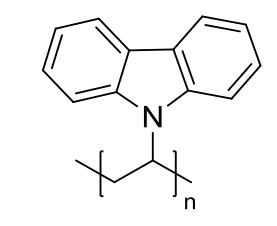

b) PVK
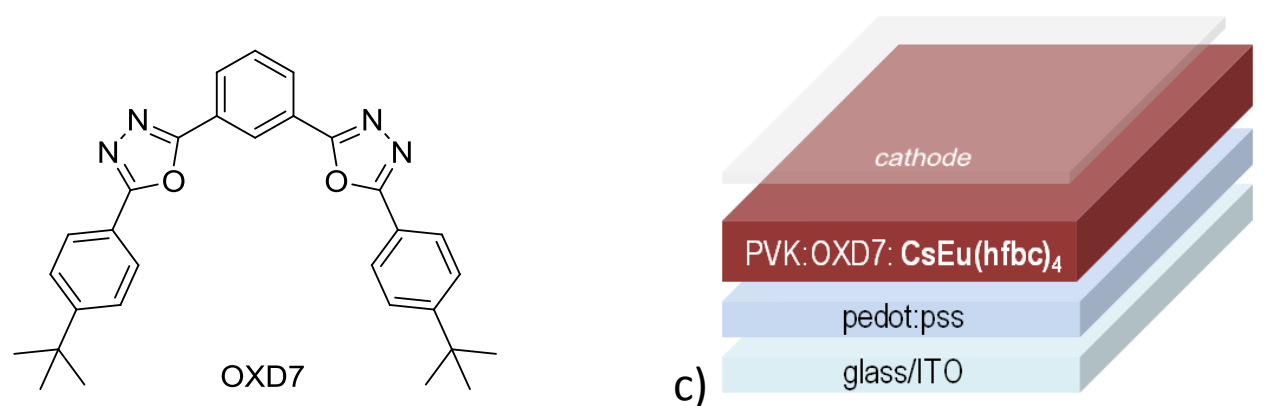

Figure 2 a) Molecular structure of the two enantiomers of $\mathrm{CsEu}(\mathrm{hfbc})_{4}$; b) PVK polymer host and OXD7 additive constituting the blend for the emitting layer; c) Device architecture showing the thin film containing the Eu(III) complex-based active layer, sandwiched between a PEDOT:PSS-coated ITO anode and a Ba/Al cathode.

a)

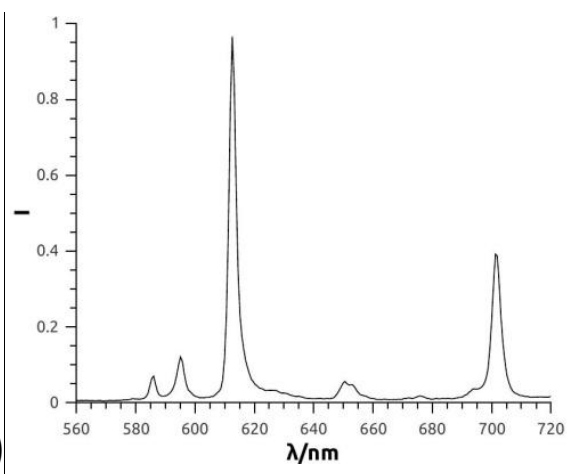

c)

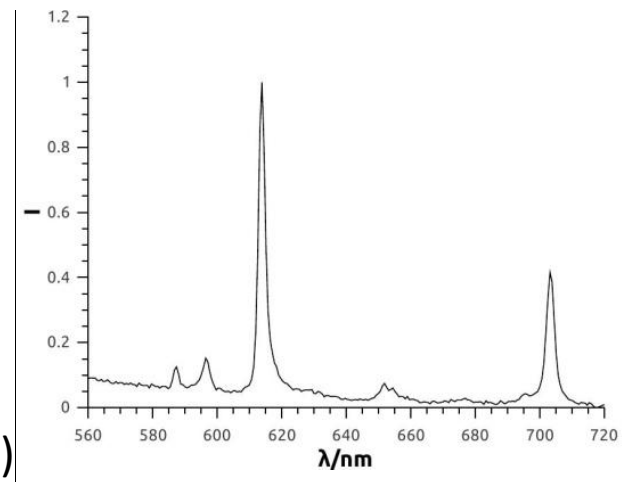

b)

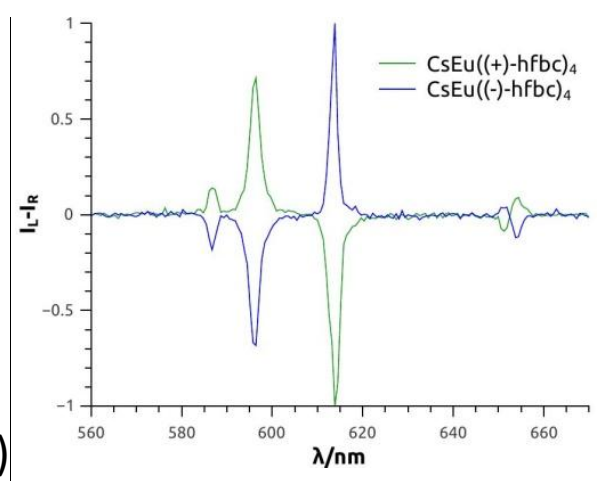

d)

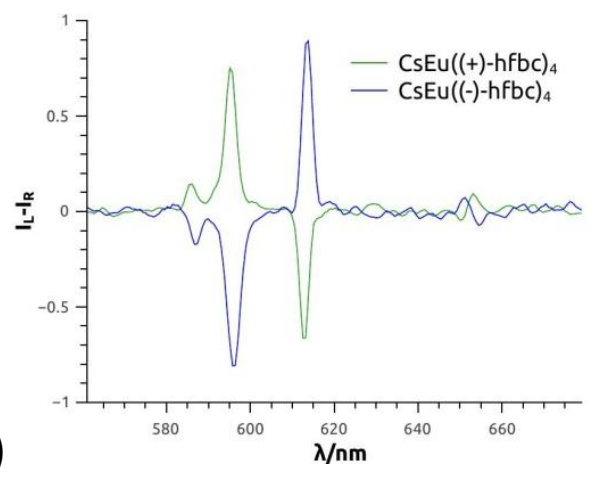

Figure 3

Photoluminescence of $\mathrm{CsEu}(\mathrm{hfbc})_{4}$ in $\mathrm{CHCl}_{3}$ solution (a) and in the blend film (c) and circularly polarized photoluminescence of (b) $\mathrm{CHCl}_{3}$ solution; d) film. 


\section{WILEY-VCH}

In our strategy for the development of the CP-OLED architecture, two critical issues were addressed: inhibition of all the emission from the matrix, in order to have light stemming only from the Eu(III)-complex, and minimization of internal reflection of emitted light on the back electrode, to preserve CPEL.

In such CP-OLEDs the emission from the matrix is undesired because all the photons arising from other sources than the chiral $\mathrm{Eu}(\mathrm{III})$ complex would reduce the chiroptical response (in terms of $g$ factor) of the system. We focused on the easily processable polyvinylcarbazole (PVK, Figure $\mathbf{2 b}$ ), possessing good hole transport properties and weak electroluminescence, which was demonstrated as a suitable host for Eu(III) complexes. ${ }^{[22]}$ In order to provide a better carrier transport and carrier/exciton confinement, a soluble oxidiazole derivative, 1,3-bis[2-(4-tert-butylphenyl)-1,3,4oxadiazo-5-yl]benzene (OXD7, Figure 2b), was added to the blend, typically in the ratio PVK:OXD7/3:1. We found that a concentration of $10 \mathrm{wt} . \%$ of the Eu(III) complex emitter in the blend was optimal to collect pure Eu(III)-centred emission, although in the PL spectrum the PVK emission was still dominant in the blue region (Figure 3c, Figure S3).

a)

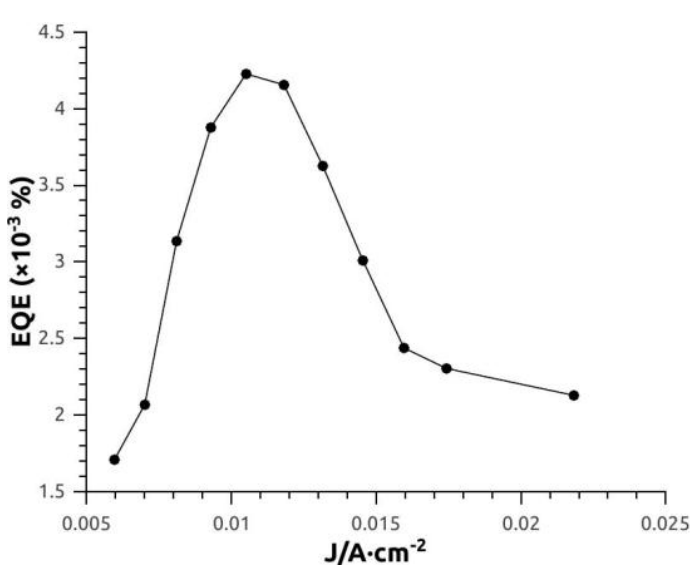

b)

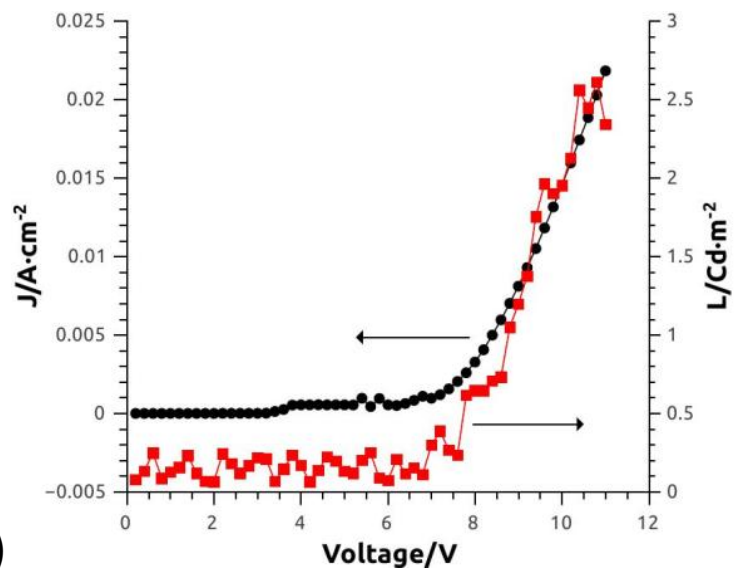

Figure 4 a) Total external quantum efficiency versus current density J; b) Representative curve of0 J (circles) and luminance L (squares) as a function of applied voltage.

The reflection of light reverses the handedness of CP light: if this occurs on the back electrode, the polarization of light exiting the ITO window would be reduced, ${ }^{[11]}$ even though the intrinsic emission of the active component in the device is strongly polarized (Figure 3b,d). The usual configuration of OLEDs consists in a fully reflecting metal top electrode, in order to collect also the photons emitted opposite to the transparent substrate. In our devices, this would be highly detrimental and therefore the reduction of the cathode thickness to obtain a semi-transparent surface is imperative. $\mathrm{A} \mathrm{Ba} / \mathrm{Al}$ cathode was preferred to the more conventional $\mathrm{LiF} / \mathrm{Al} / \mathrm{Ag},{ }^{[23]}$ commonly used in top-emitting devices, since it was demonstrated as a good electrode in PVK-based 


\section{WILEY-VCH}

devices. ${ }^{[20]}$ A $50 \%$ cathode transmittance was found to be suitable to grant reduced reflection, thus ensuring a highly polarized EL with a good stability.

Following this strategy, we fabricated devices with a very basic architecture: on top of PEDOT:PSS covered glass/ITO substrate, a CsEu(hfbc) $)_{4}$ 10wt.\%-doped PVK:OXD7 single active layer was deposited by spin-coating technique (Figure 2c). The appropriate solvent and concentration of the solution ensured good film quality (Figure S10). Devices with $4 \mathrm{~nm} \mathrm{Ba}$ and different Al cathode thickness in the range from $80 \mathrm{~nm}$ to $6 \mathrm{~nm}(50 \%$ transmittance at $612 \mathrm{~nm})$ were built to investigate the effect of internal reflections on the $g$-factor. The maximum EQE, measured as the total number of emitted photons on number of injected carriers, was $4.2 \cdot 10^{-3} \%$ (current efficiency $5 \cdot 10^{-3} \mathrm{Cd} / \mathrm{A}$, power efficiency $6.7 \cdot 10^{-3} \mathrm{~lm} / \mathrm{W}$, Figure 4). Devices switched on at about $8 \mathrm{~V}$ and were driven at 20 $\mathrm{mA} / \mathrm{cm}^{2}$ (about $10 \mathrm{~V}$ ) for recording CPEL spectra. The light was collected from the glass surface in perpendicular direction and the stability of the emission spectra was verified for the time required for the characterization (Figure S6).

The total EL spectrum (Figure 5a) showed the typical Eu(III)-centred emission in a wide range of driving voltages (Figure S4).

The CPEL was measured by using a quarter wave retarder and a linear polarizer prior to a CCD detector. By rotating the easy axis of the quarter wave plate by $\pm 45^{\circ}$ with respect to the linear polarizer, the $I_{L}$ and $I_{R}$ polarized components of the emission were alternatively acquired. By subtracting the $\mathrm{I}_{\mathrm{L}}$ and $\mathrm{I}_{\mathrm{R}}$ spectra, the CPEL spectra were obtained; $g_{\mathrm{EL}}$ was derived according to the definition reported above.

The CPEL spectra obtained for the devices containing the two enantiomers $\left(\mathrm{CsEu}((+)-\mathrm{hfbc})_{4}\right.$ and $\left.\mathrm{CsEu}((-)-\mathrm{hfbc})_{4}\right)$, were almost perfectly mirror image (Figure 5b) and showed the same patterns of bands reported for the CP PL spectra measured in $\mathrm{CHCl}_{3}$ (Figure 3b). ${ }^{[21]}$ This excludes all artifacts due to linear polarization and birefringence of any component in both OLEDs and detection apparatus. It further ensures that the observed CPEL is Eu(III)-centered, ruling out any contribution of the polymeric matrix to the measured EL and CPEL.

A $g_{\text {EL }}$ value of about \pm 0.75 , at $595 \mathrm{~nm}$ (for the $\mathrm{Eu}^{5} \mathrm{D}_{0} \rightarrow{ }^{7} \mathrm{~F}_{1}$ transition, see Table 1), was recorded from the devices with the thinnest cathode ( $4 \mathrm{~nm} \mathrm{Ba+6} \mathrm{nm} \mathrm{Al);} \mathrm{this} \mathrm{means} \mathrm{that} \mathrm{about} 70 \%$ of the EL is circularly polarized. To the best of our knowledge, this is the highest value ever measured for a CP-OLED and the very first report of lanthanide-centered circularly polarized EL. 


\section{WILEY-VCH}

Similarly, the CP EL of the band at $612 \mathrm{~nm}$ (for the $\mathrm{Eu}^{5} \mathrm{D}_{0} \rightarrow{ }^{7} \mathrm{~F}_{2}$ transition, see Table 1) showed a value of $g_{\mathrm{EL}}$ of $-0.09 /+0.15$. As expected from the PL spectrum, this band possessed an opposite sign with respect to the emission at $595 \mathrm{~nm}$. The measurements carried out on devices with different cathode thickness showed an exponential-like reduction of $g_{\mathrm{EL}}$ upon increasing electrode thickness (Figure 6). The total EQE does not vary significantly with the cathode thickness since all the emitted photons are collected (both forward and backward emission, see Table S2), as also demonstrated by Lee et al. ${ }^{[24]}$

a)

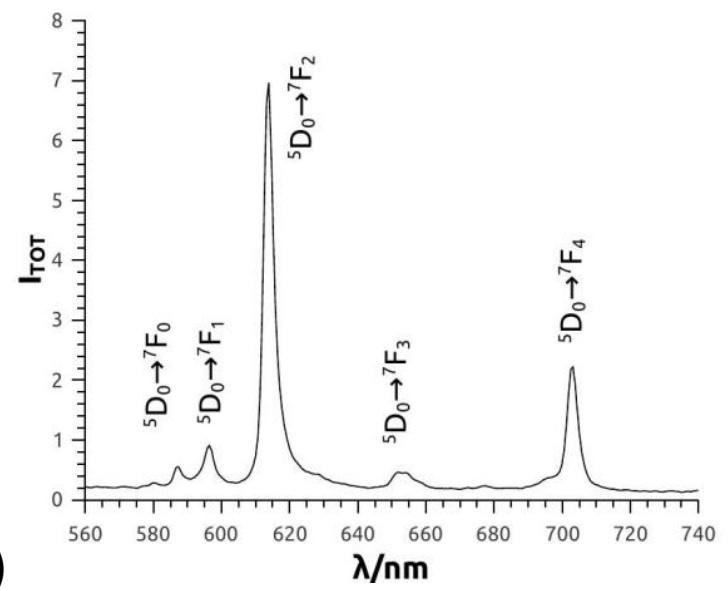

b)

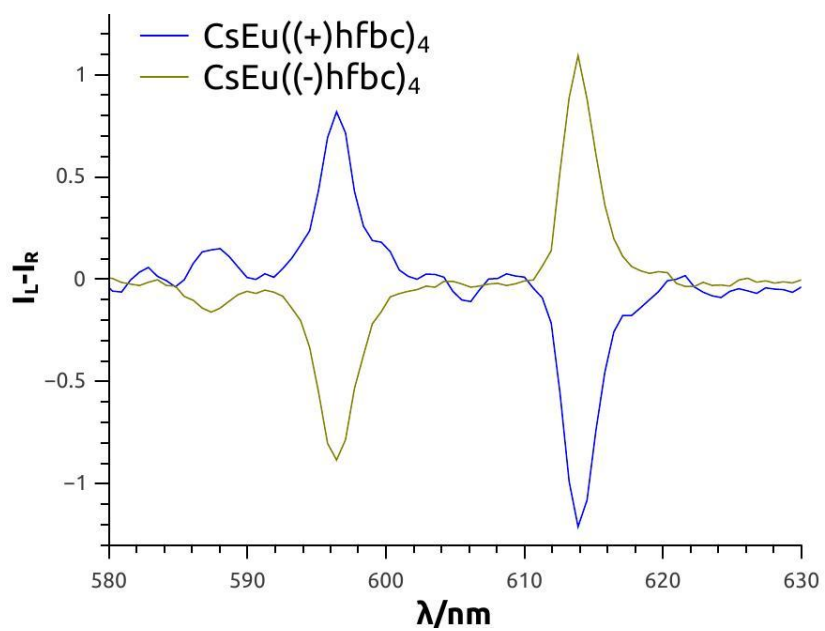

Figure 5 a) EL spectrum of $\mathrm{CsEu}(\mathrm{hfbc})_{4}$-based OLEDs; b) CPEL of the devices containing each enantiomer of $\mathrm{CsEu}(\mathrm{hfbc})_{4}$.

Table 1 EL dissymmetry factors $\left(g_{\mathrm{EL}}\right)$ for $\mathrm{CsEu}(( \pm) \text {-hfbc })_{4}$-based devices with standard deviations $\left(\sigma_{\mathrm{d}}\right)$ and PL dissymmetry factors $\left(g_{\mathrm{PL}}\right)$ of the complexes in $\mathrm{CHCl}_{3}$ solution.

\begin{tabular}{lll}
\hline & $595 \mathrm{~nm}\left({ }^{5} \mathrm{D}_{0} \rightarrow{ }^{7} \mathrm{~F}_{1}\right)$ & $612 \mathrm{~nm}\left({ }^{5} \mathrm{D}_{0} \rightarrow{ }^{7} \mathrm{~F}_{2}\right)$ \\
\hline $\mathrm{CsEu}((+)-\mathrm{hfbc})_{4} g_{\mathrm{EL}}\left(\sigma_{\mathrm{d}}\right)$ & $+0.73(0.08)$ & $-0.09(0.05)$ \\
$\mathrm{CsEu}((-)-\mathrm{hfbc})_{4} g_{\mathrm{EL}}\left(\sigma_{\mathrm{d}}\right)$ & $-0.79(0.02)$ & $+0.15(0.06)$ \\
$\mathrm{CsEu}((+)-\mathrm{hfbc})_{4} \mathrm{~g}_{\mathrm{PL}}\left(\sigma_{\mathrm{d}}\right)$ & $+1.41(0.04)$ & $-0.25(0.04)$ \\
$\mathrm{CsEu}((-)-\mathrm{hfbc})_{4} \mathrm{~g}_{\mathrm{PL}}\left(\sigma_{\mathrm{d}}\right)$ & $-1.41(0.05)$ & $+0.22(0.04)$ \\
\hline
\end{tabular}




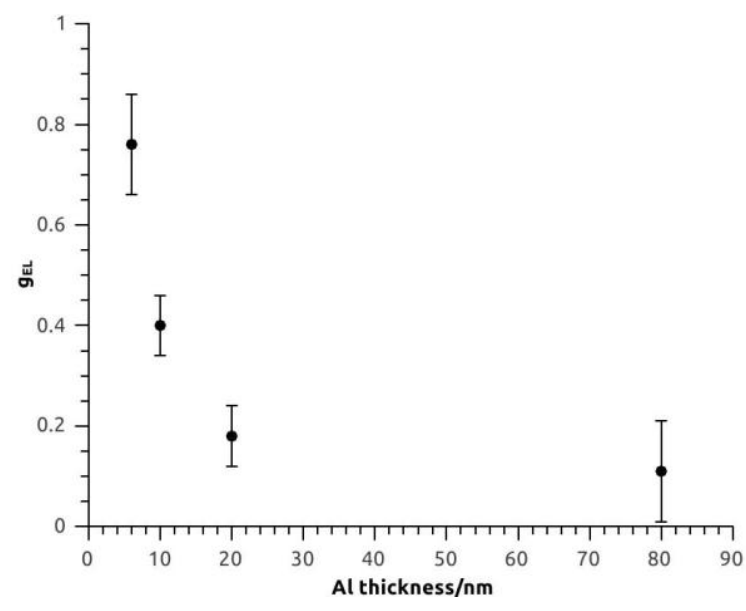

Figure 6 Four experimental $\mathrm{g}_{\mathrm{EL}}$ values obtained for the transition at $595 \mathrm{~nm}$ as a function of the cathode thickness. The error bars represent \pm 2 standard deviations. We refer to the SI for a possible explanations of this trend.

In conclusion, we have presented a novel approach for manufacturing OLEDs with intrinsic sharp $\mathrm{CP}$ emission. For the first time a chiral lanthanide complex was successfully used as the direct circularly polarized emitter in a well-established solution-processed device and an unprecedented study of the effect of cathode transmittance on the level of CPEL was carried out. Following our strategy, we manufactured devices that outperform known CP-OLEDs by at least a factor 2 in terms of $g_{\mathrm{EL}}$ value. The results disclosed herein represent a proof-of-concept and there is plenty of room for improvement, for example by rational molecular design of chiral Ln(III) complexes with higher PL quantum yield, and also for extension to lanthanide complexes emitting in other spectral regions, including the near infrared. We believe that this may open up new perspectives for applications of rare earth elements in chiral optoelectronics and photonics.

\section{Methods}

A blend of PVK:OXD7:CsEu(hfbc $)_{4}$ in the mass ratio (3:1:0.4) was dissolved in $\mathrm{CHCl}_{3} 15 \mathrm{mg} / \mathrm{mL}$. Indium tin oxide (ITO; $15 \Omega$ per square) substrates were cleaned by sonication in distilled water, acetone, and isopropyl alcohol. After treatment with nitrogen plasma, a thin film $(40 \mathrm{~nm})$ of filtered (nylon $0.45 \mu \mathrm{m})$ poly-(3,4-ethylenedioxythiophene)poly(styrenesulfonic acid) (PEDOT:PSS, Clevios P VP AI 4083, H.C. Starck) used as a hole-injection layer at the anode interface was spin-coated onto the ITO substrate, and then dried under nitrogen atmosphere at $150{ }^{\circ} \mathrm{C}$ for 15 minutes. A film of emitting blend $(65-120 \mathrm{~nm})$ was then spin-coated. A thin layer of barium (4 nm) and subsequently a layer of Al $(6-80 \mathrm{~nm})$ were deposited on the top by vacuum $\left(5 \times 10^{-7}\right.$ mbar $)$ thermal evaporation. The device active area was $5.4 \mathrm{~mm}^{2}$. All operations were carried out inside a nitrogen-filled drybox with less than 0.1 ppm of both oxygen and water. 


\section{WILEY-VCH}

CPEL spectra were recorded using Spex 270M monochromator combined with CCD, using a linear polarizer and a THORLABS achromatic $(400-800 \mathrm{~nm})$ quarter wave plate prior to the detector.

\section{Acknowledgements}

Financial support from MIUR-PRIN 2012A4Z2RY is acknowledged. U.G acknowledges support from Cariplo Foundation (2012-0844). Ettore Castiglioni is gratefully thanked for the help in the initial setup design for measuring CPEL.

\section{Supporting Information}

Supporting Information is available from the Wiley Online Library or from the author.

\section{References}

[1] S. Reineke, M. Thomschke, B. Lüssem, K. Leo, Rev. Mod. Phys. 2013, 85, 1245.

[2] M. Grell, D. D. Bradley, Adv. Mater. 1999, 11, 895; A. Bolognesi, C. Botta, D. Facchinetti, M. Jandke, K. Kreger, P. Strohriegl, A. Relini, R. Rolandi, S. Blumstengel, Adv. Mater. 2001, 13, 1072.

[3] B. L. Feringa, Acc. Chem. Res. 2001, 34, 504.

[4] C. Wang, H. Fei, Y. Qiu, Y. Yang, Z. Wei, Y. Tian, Y. Chen, Y. Zhao, Appl. Phys. Lett. 1999, 74, 19.

[5] C. Wagenknecht, C.-M. Li, A. Reingruber, X.-H. Bao, A. Goebel, Y.-A. Chen, Q. Zhang, K. Chen, J.-W. Pan, Nat Photon 2010, 4, 549.

[6] R. Farshchi, M. Ramsteiner, J. Herfort, A. Tahraoui, H. T. Grahn, Appl. Phys. Lett. 2011, 98, 162508.

[7] a) Y. Yang, R. C. da Costa, M. J. Fuchter, A. J. Campbell, Nature Photon. 2013, 7, 634;

b) W. Sparks, J. Hough, L. Kolokolova, T. Germer, F. Chen, S. DasSarma, P. DasSarma, F. Robb, N. Manset, I. Reid, J. Quant. Spetroscop. Ra. 2009, 110, 1771.

[8] T. Novikova, A. Pierangelo, A. De Martino, A. Benali, P. Validire, Opt. Photonics News 2012.

[9] M. Oda, H. G. Nothofer, G. Lieser, U. Scherf, S. C. J. Meskers, D. Neher, Adv. Mater. 2000, $12,362$.

[10] F. Zinna, L. Di Bari, Chirality 2014, 10.1002/chir.22382.

[11] E. Peeters, M. P. T. Christiaans, R. A. J. Janssen, H. F. M. Schoo, H. P. J. M. Dekkers, E. W. Meijer, J. Am. Chem. Soc. 1997, 119, 9909.

[12] Y. Geng, A. Trajkovska, S. W. Culligan, J. J. Ou, H. M. P. Chen, D. Katsis, S. H. Chen, J. Am. Chem. Soc. 2003, 125, 14032.

[13] G. Pescitelli, L. Di Bari, N. Berova, Chem. Soc. Rev. 2014, 43, 5211.

[14] Y. Yang, R. C. da Costa, D.-M. Smilgies, A. J. Campbell, M. J. Fuchter, Adv. Mater. 2013, 25, 2624.

[15] A. de Bettencourt-Dias, Dalton Trans. 2007, 2229.

[16] J. Kido, Y. Okamoto, Chem. Rev, 2002, 102, 2357; Z. Chen, F. Ding, F. Hao, M. Guan, Z. Bian, B. Ding, C. Huang, New J. Chem. 2010, 34, 487.

[17] S. V. Eliseeva, J.-C. G. Bunzli, Chem. Soc. Rev. 2010, 39, 189.

[18] T. Canzler, J. Kido, Org. Electron. 2006, 7, 29.

[19] S. Zhang, G. A. Turnbull, I. D. W. Samuel, Org. Electron. 2012, 13, 3091. 


\section{WILEY-VCH}

[20] U. Giovanella, M. Pasini, C. Freund, C. Botta, W. Porzio, S. Destri, J. Phys. Chem. C 2009, 113, 2290.

[21] J. L. Lunkley, D. Shirotani, K. Yamanari, S. Kaizaki, G. Muller, Inorg. Chem. 2011, 50, 12724.

[22] a) Y. Zhang, C. Li, H. Shi, B. Du, W. Yang, Y. Cao, New J. Chem. 2007, 31, 569; b) C. Rothe, L. O. Pålsson, A. P. Monkman, Chem. Phys. 2002, 285, 95.

[23] J. W. Huh, J. Moon, J. W. Lee, J. Lee, D.-H. Cho, J.-W. Shin, J.-H. Han, J. Hwang, C. W. Joo, J.-I. Lee, H. Y. Chu, Org. Electron. 2013, 14, 2039.

[24] C.-J. Lee, D.-G. Moon, Trans. Electr. Electron. Mater. 2014, 15, 45.

Received: ((will be filled in by the editorial staff))

Revised: ((will be filled in by the editorial staff))

Published online: ((will be filled in by the editorial staff)) 


\section{WILEY-VCH}

The first Circularly polarized OLED (CP-OLED) based on a chiral lanthanide complex is developed. With a simple architecture, this proof-of-concept device attains the highest polarization reported so far and additionally presents the first example of Ln-based OLED that can be turned into a CP-OLED, fully retaining the special features of Ln-based OLEDs.

Francesco Zinna; Umberto Giovanella; Lorenzo Di Bari*

Highly Circularly Polarized Electroluminescence from a Chiral Europium Complex

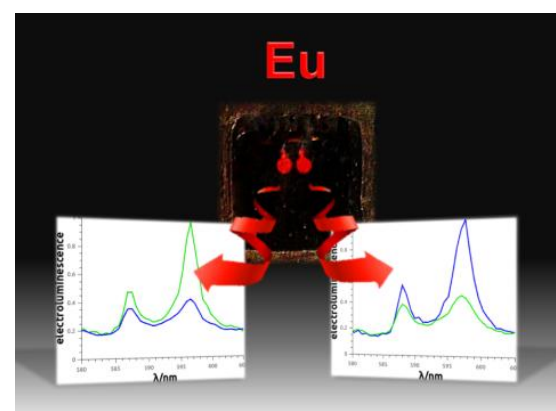




\section{WILEY-VCH}

Copyright WILEY-VCH Verlag GmbH \& Co. KGaA, 69469 Weinheim, Germany, 2013.

\section{Supporting Information}

\section{Highly Circularly Polarized Electroluminescence from a Chiral Europium Complex}

Francesco Zinna; Umberto Giovanella; Lorenzo Di Bari ${ }^{*}$

\section{Synthesis of the complexes $\operatorname{CsEu}(( \pm)-h f b c)_{4}$}

The complexes were synthesized following a slightly modified literature procedure, ${ }^{[\mathrm{S} 1]}$ described below. Their identity and purity was confirmed by comparing ${ }^{1} \mathrm{H}-\mathrm{NMR}$ (Agilent Inova 600 operating at $14.1 \mathrm{~T})$ spectra with the literature data.

\section{Synthesis of $\mathrm{CsEu}((+)-h f b c)_{4}$}

$\mathrm{CsCl}(200 \mathrm{mg}, 1.01 \mathrm{mmol})$ and $\mathrm{EuCl}_{3}(132 \mathrm{mg}, 0.50 \mathrm{mmol})$ were dissolved in $\mathrm{H}_{2} \mathrm{O}(20 \mathrm{~mL})$. In the mean time a solution containing (+)-heptafluorobutyrylcamphor ((+)-hfbcH; $0.54 \mathrm{~mL}, 2.04 \mathrm{mmol})$ and triethylamine $(0.30 \mathrm{~mL}, 2.15 \mathrm{mmol})$ in $\mathrm{CHCl}_{3}(30 \mathrm{~mL})$ was prepared. The two solutions were mixed together. The resulting biphasic system was left for 1 hour at room temperature under vigorous stirring.

The $\mathrm{CHCl}_{3}$ layer was recovered and the aqueous phase was extracted with fresh $\mathrm{CHCl}_{3}(2 \times 15 \mathrm{~mL})$. The combined organic phases were washed with water $(3 \mathrm{x} 15 \mathrm{~mL})$, dried over anhydrous $\mathrm{Na}_{2} \mathrm{SO}_{4}$ and the solvent was removed under vacuum. The crude product was crystallized from hot $\mathrm{CH}_{3} \mathrm{CN}$.

The title compound was obtained as white needle crystals in $21 \%$ yield.

${ }^{1} \mathrm{H}-\mathrm{NMR}\left(\mathrm{CDCl}_{3}, 600 \mathrm{MHz}, 25.0{ }^{\circ} \mathrm{C}\right.$, broad singlets), $\delta$ (ppm): $5.20\left(\mathrm{Me}^{3}\right) ;-0.89\left(\mathrm{Me}^{2}\right)$; -0.99 (A); $1.66(\mathrm{C}) ;-2.72\left(\mathrm{Me}^{1}+\mathrm{D}\right) ;-4.55(\mathrm{E}) ;-6.35(\mathrm{~B})$.

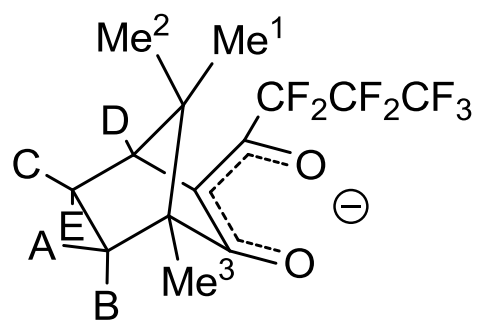




\section{WILEY-VCH}

\section{Synthesys of $\operatorname{CsEu}((-)-h f b c)_{4}$}

For the synthesis of $\mathrm{CsEu}((-)-\mathrm{hfbc})_{4}$, the same procedure reported above for $\mathrm{CsEu}((+)-\mathrm{hfbc})_{4}$ was followed. Yield 23\%; same ${ }^{1} \mathrm{H}$ NMR data as above.

\section{CPL measurements}

The instrumental set-up for detection of circular polarization of emitted light consisted in a Spex 270M monochromator combined with CCD. Between the sample and the detector we put a quarter wave plate (Thorlabs, AQWP05M-600) which was rotated through angles defining the orientation of the easy axis vs. the linear polarizer (Oriel 25010), which was kept fix.

\section{Setup calibration}

We validated the CPL measurements using $\mathrm{CHCl}_{3}$ solutions $\left(2 \cdot 10^{-3} \mathrm{M}, \lambda_{\text {exc }}=365 \mathrm{~nm}\right)$ of each $\mathrm{CsEu}(\mathrm{hfbc})_{4}$ enantiomer. The emission of each solution was measured by rotating the quarter wave plate by $\pm 45^{\circ}$; the measurements were repeated 6 times for each equivalent position of the quarter wave plate for each solution. The CPL spectrum (Figure 3b) was obtained by subtracting the left and right component of the emission $\left(\mathrm{I}_{\mathrm{L}}-\mathrm{I}_{\mathrm{R}}\right)$; the $g_{\mathrm{PL}}-\mathrm{S}$ were calculated using the definition:

$g_{\mathrm{PL}}=2 \frac{\mathrm{I}_{\mathrm{L}}-\mathrm{I}_{\mathrm{R}}}{\mathrm{I}_{\mathrm{L}}+\mathrm{I}_{\mathrm{R}}}$

averaging over the whole set of measurements. The total intensity $\left(\mathrm{I}_{\mathrm{L}}+\mathrm{I}_{\mathrm{R}}\right)$ was corrected by subtracting the background (the background signal was generally below $5 \%$ of the least intense band). The CPL spectra obtained for the two enantiomers were mirror-like, as expected. This rules out any linear birefringence contribution in the measurement apparatus. The calculated $g_{\mathrm{PL}}$ values were in very good agreement with the ones reported in the literature ${ }^{[\mathrm{S} 2]}$ for $\mathrm{CsEu}((+)-\mathrm{hfbc})_{4}$ (see Table S1).

Table S1 Photoluminescence dissymmetry factors $\left(\mathrm{g}_{\mathrm{PL}}\right)$ for $\mathrm{CsEu}(( \pm)-\mathrm{hfbc})_{4}$ solutions $\mathrm{CHCl}_{3}$ with standard deviations $\left(\sigma_{\mathrm{d}}\right)$ and the $\mathrm{g}_{\mathrm{PL}}$ values reported in the literature ${ }^{[\mathrm{S} 2]}$ for $\mathrm{CsEu}((+)-\mathrm{hfbc})_{4}$.

\begin{tabular}{lll}
\hline & $595 \mathrm{~nm}\left({ }^{5} \mathrm{D}_{0} \rightarrow{ }^{7} \mathrm{~F}_{1}\right)$ & $612 \mathrm{~nm}\left({ }^{5} \mathrm{D}_{0} \rightarrow{ }^{7} \mathrm{~F}_{2}\right)$ \\
\hline $\mathrm{CsEu}((+)-\mathrm{hfbc})_{4} \mathrm{~g}_{\mathrm{PL}}\left(\sigma_{\mathrm{d}}\right)$ & $+1.41(0.04)$ & $-0.25(0.04)$ \\
$\mathrm{CsEu}((-)-\mathrm{hfbc})_{4} \mathrm{~g}_{\mathrm{PL}}\left(\sigma_{\mathrm{d}}\right)$ & $-1.41(0.05)$ & $+0.22(0.04)$ \\
$\mathrm{CsEu}((+)-\mathrm{hfbc})_{4} \mathrm{~g}_{\mathrm{PL}}[\mathrm{S} 2]$ & +1.38 & -0.23 \\
\hline
\end{tabular}




\section{WILEY-VCH}

\section{UV and ECD spectra of CsEu(hfbc $)_{4}$}
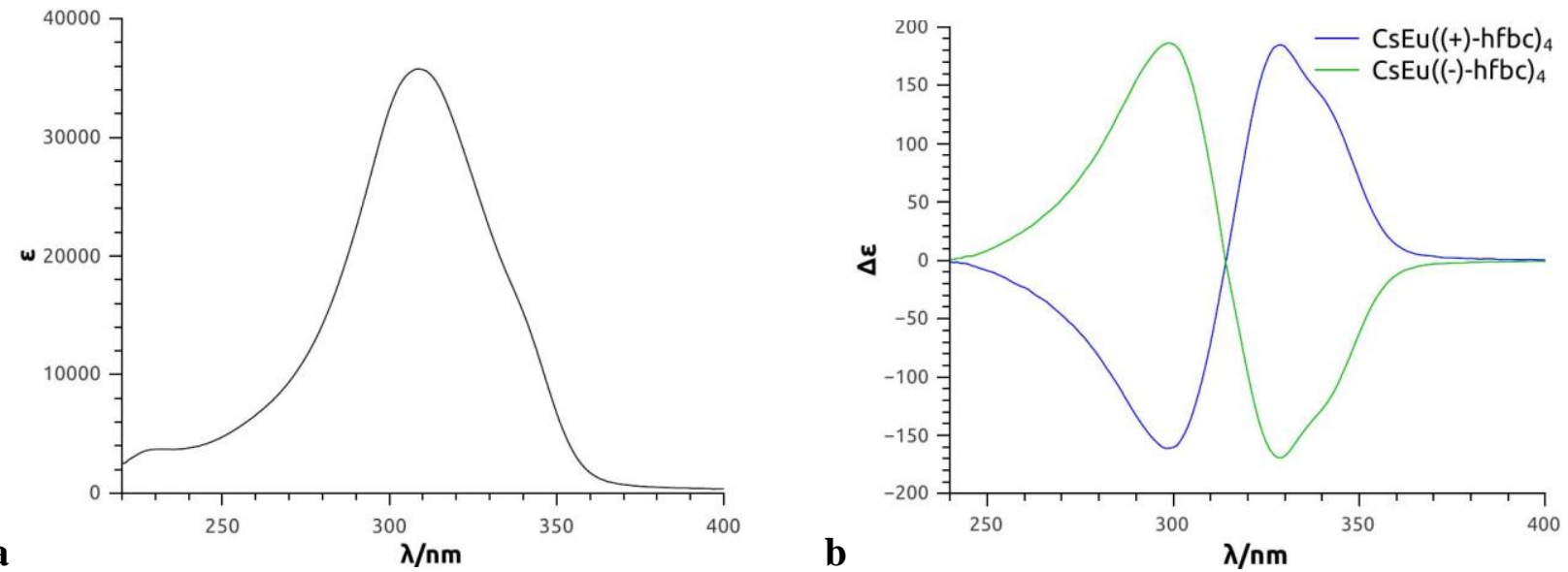

Figure S1 Absorption spectrum (a) and electronic circular dichroism (b) for $\mathrm{CsEu}(\mathrm{hfbc})_{4}$ in $\mathrm{CHCl}_{3}$ solution.

\section{Photophysical characterization of the active layer film}
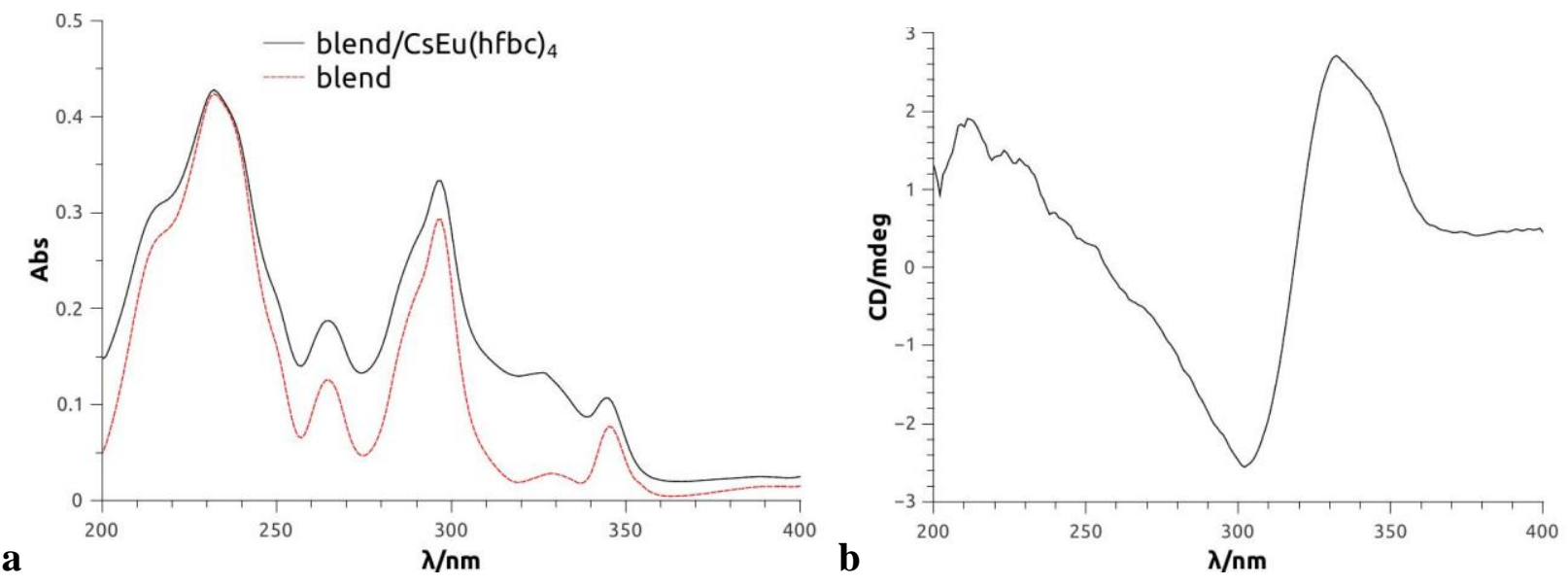

Figure S2 a, absorption spectrum for the active layer film with (black) and without the complex (red). b, electronic circular dichroism for the blend film with $\mathrm{CsEu}((-)-\mathrm{hfbc})_{4}$.

\section{PL and EL measurements on the film}

The photoluminescence spectrum of the film shows the emission of both the Eu(III) species and PVK (Figure S3), while PVK contribution to the emission is almost completely quenched in electroluminescence even at high driving voltages (Figure S4). 


\section{WiLEY-VCH}

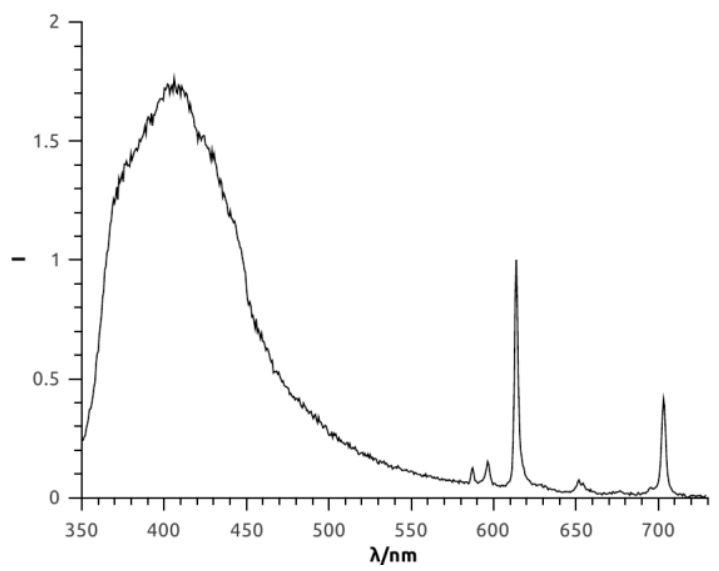

Figure S3

Total photoluminescence of the blend film.

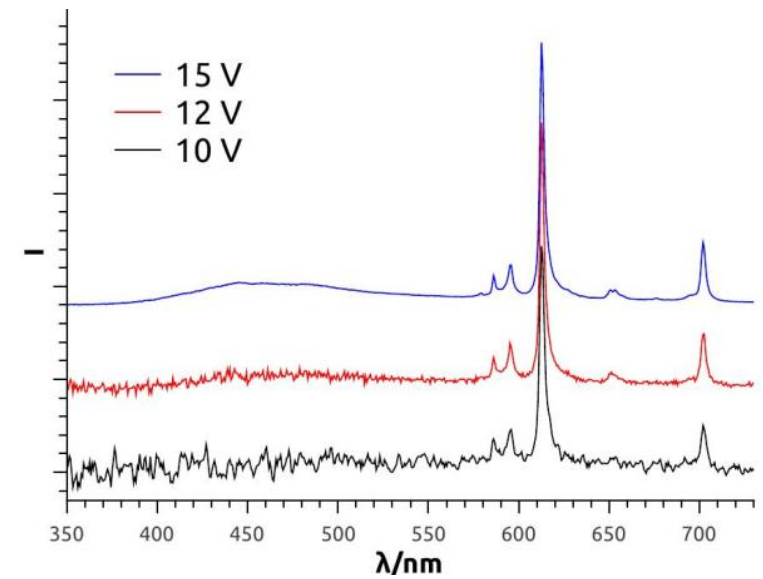

Figure S4 Voltage dependence of EL spectra of a ITO/PEDOT:PSS/PVK:OXD7: CsEu((-)-hfbc) $4 / \mathrm{Ba} / \mathrm{Al}$ device (10-15 V). Spectra are vertically shifted for clarity.

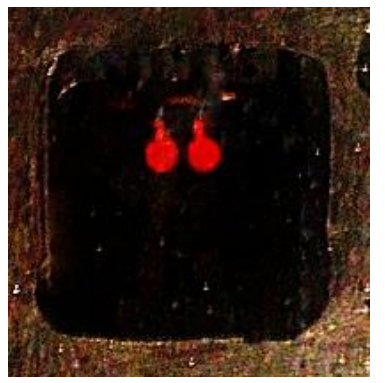

Figure S5 Picture of the device, two pixels are switched on. 


\section{WILEY-VCH}

\section{CPEL measurements on the LEDs}

The devices were turned on by applying 10-12 V (corresponding to $20 \mathrm{~mA} / \mathrm{cm}^{2}$ ) and were let stabilize for several seconds (15-20 s) before the measurement. The differential emission was measured by rotating the quarter wave plate by $\pm 45^{\circ}$, as described above for the solutions. The measurements were repeated at least 4 times for each equivalent position of the quarter wave plate for each LED pixel. This set of measurements was repeated at least on 5 pixels for each device. The

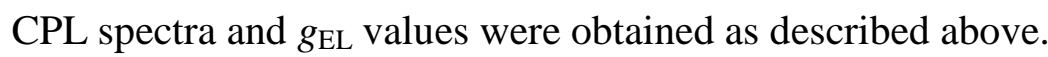
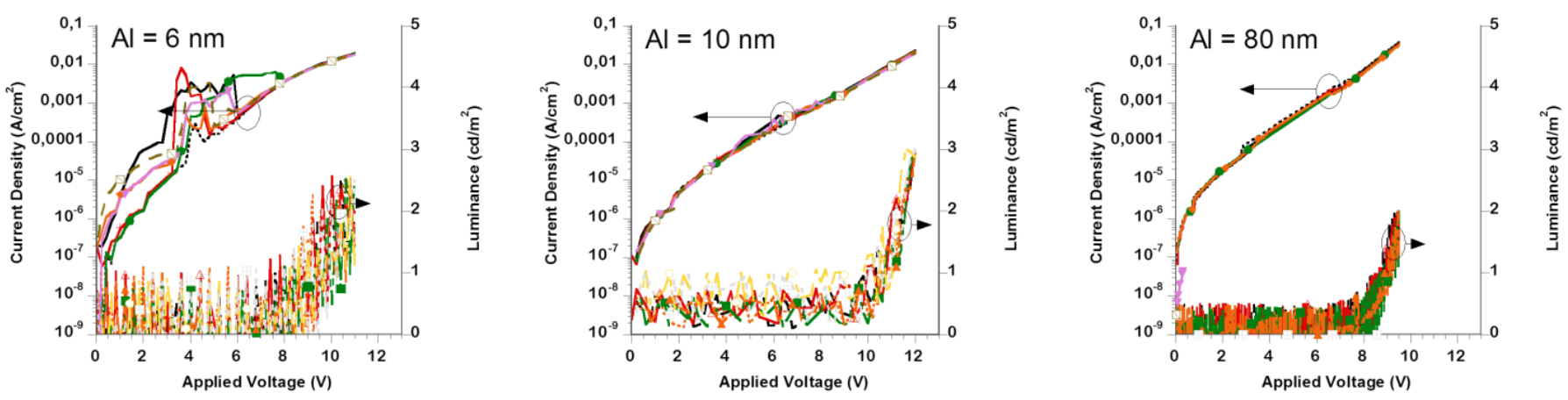

Figure S6 Reproducibility (8 sweeps) of current density-brightness-voltage curves of ITO/PEDOT:PSS/PVK:OXD7: $\mathrm{CsEu}((+)-\mathrm{hfbc})_{4} / \mathrm{Ba} / \mathrm{Al}$ devices with $6 \mathrm{~nm}$ (left), $10 \mathrm{~nm}$ (center) and $80 \mathrm{~nm} \mathrm{Al}$ cathode (right).

Effect of the active layer thickness and the blend ratio on the electroluminescence dissymmetry factor

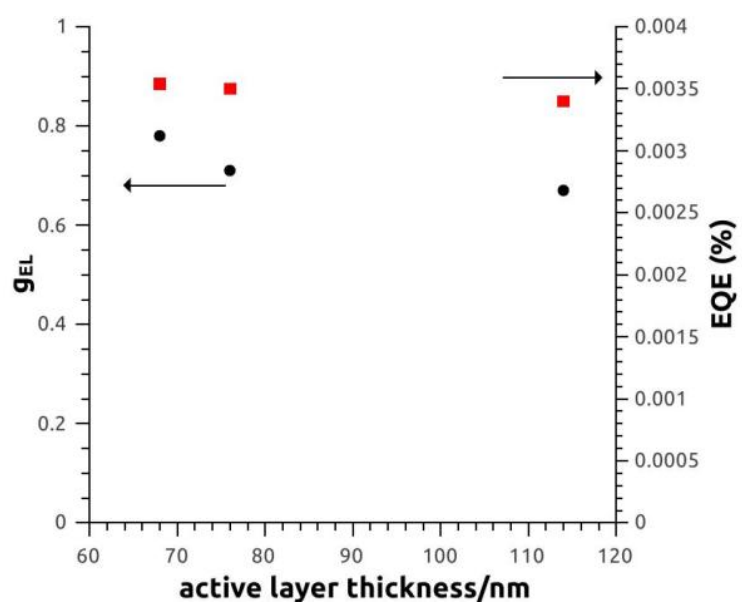

Figure S7 Total external quantum efficiency (EQE) and $g_{\mathrm{EL}}$ of three devices with varying active layer thickness. The cathode thickness $(\mathrm{Ba} / \mathrm{Al} 4 / 6 \mathrm{~nm})$ and the blend ratio $\left(10 \% \mathrm{w} / \mathrm{w} \mathrm{CsEu}(\mathrm{hfbc})_{4}\right)$ were kept constant. 


\section{WILEY-VCH}

Figure S7 shows that $g_{\mathrm{EL}}$ factors do not vary significantly with the active layer thickness, moreover a blend with $20 \%$ w/w of $\mathrm{CsEu}(\mathrm{hfbc})_{4}$ showed a similar $g_{\mathrm{EL}}$ value $(0.71)$.

\section{Reflection on the cathode}

As discussed in the text, the reflection of the emitted light on the cathode is a major issue for the polarization performances of CP-OLED since the handedness of polarized light is reversed upon reflection. In a very simplified model we may consider that about $50 \%$ of the emitted light is directed backward towards the cathode and it is reflected. If $\mathrm{R}$ is the reflectivity of the $\mathrm{Al}$ coating, we get that the two polarized components exiting the device $\left(\mathrm{I}_{\mathrm{L}}^{\prime}\right.$ and $\left.\mathrm{I}_{\mathrm{R}}^{\prime}\right)$ are:

$\mathrm{I}_{\mathrm{L}}^{\prime}=\mathrm{I}_{\mathrm{L}}+\mathrm{R} \cdot \mathrm{I}_{\mathrm{R}}$

$I_{R}^{\prime}=I_{R}+R \cdot I_{L}$

By the definition, we get that the measured $g_{\mathrm{EL}}$ for the device is:

$g_{\mathrm{EL}}=2 \frac{\left(\mathrm{I}_{\mathrm{L}}^{\prime}-\mathrm{I}_{\mathrm{R}}^{\prime}\right)}{\left(\mathrm{I}_{\mathrm{L}}^{\prime}+\mathrm{I}_{\mathrm{R}}^{\prime}\right)}$

Substituting eq. (S2a) and (S2b) in eq. (S3), we get:

$g_{\mathrm{EL}}=g_{\mathrm{EL}}^{(0)} \frac{(1-\mathrm{R})}{(1+\mathrm{R})}$

Where $g_{\mathrm{EL}}^{(0)}$ is the intrinsic dissymmetry factor of the chiral europium species.

Hass and Waylonis ${ }^{\left[{ }^{[3]}\right.}$ measured the reflectivity $\mathrm{R}$ of $\mathrm{Al}$ thin layers for various thicknesses (Figure S8).

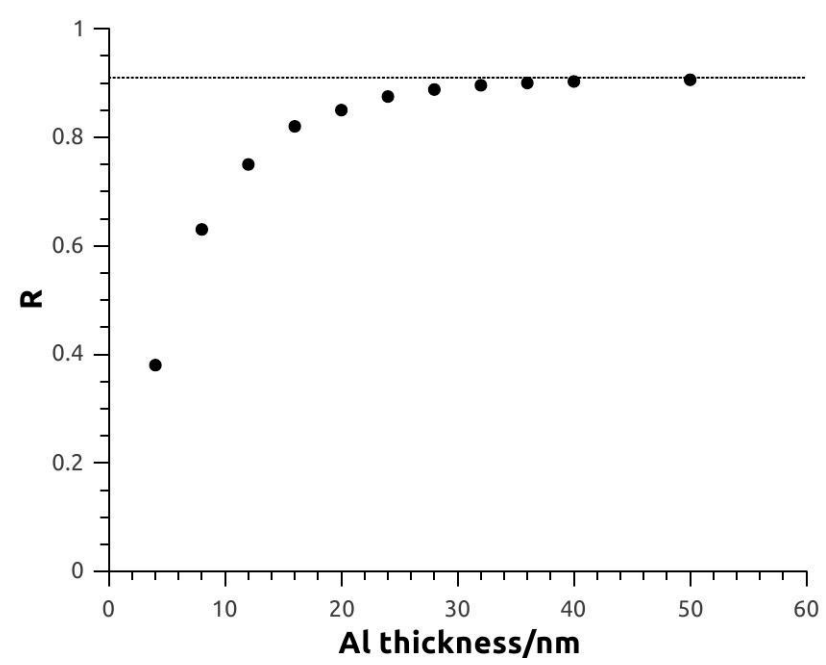




\section{WILEY-VCH}

Using these data we may calculate the expected $g_{\mathrm{EL}}$ values as a function of the $\mathrm{Al}$ cathode thickness of our devices from eq. (S4) with $g_{\mathrm{EL}}^{(0)}=1.38$. In Figure $\mathrm{S} 9$ we report these values along with our experimental points.

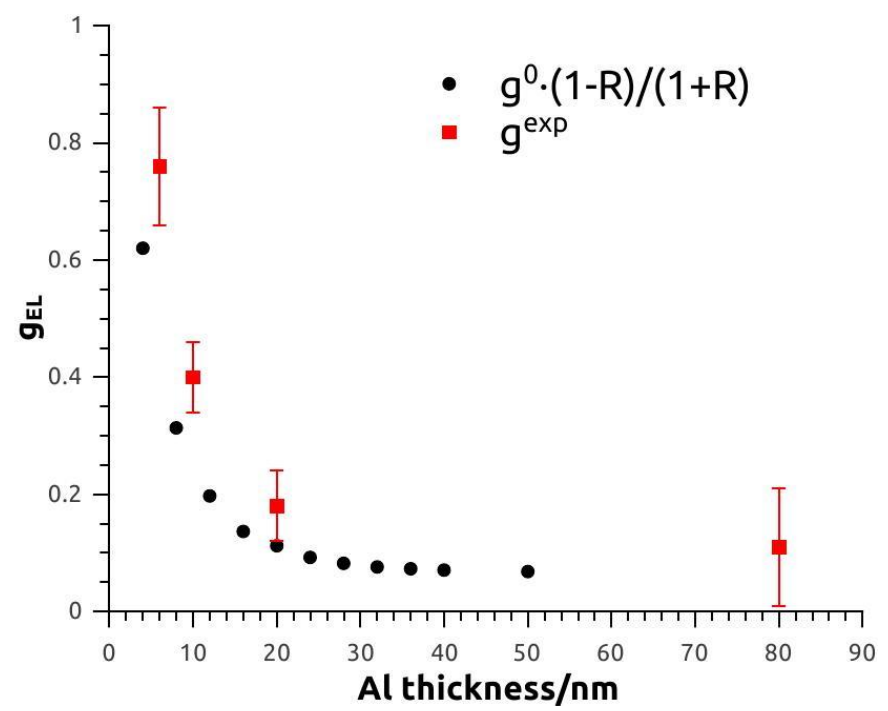

Figure S9 $\quad g_{\mathrm{EL}}$ values calculated using R by Hass and Waylonis ${ }^{[\mathrm{S} 3]}$ and eq. (S4) as a function of cathode thickness (black circles) and experimental points (red squares) obtained at $595 \mathrm{~nm}$. The error bars represent 2 standard deviations.

Figure $\mathrm{S} 8$ shows that $\mathrm{R}$ reaches a limiting value of about 0.91 and therefore eq. (S4) predicts a limiting value of 0.07 , experimentally we found a value of about 0.1 for an Al thickness of $80 \mathrm{~nm}$ (Figure S9).

Of course these considerations are solely intended to heuristically correlate the experimental trend that we observed with simple experimental values. We do not aim here to advance a comprehensive model which would require knowing several other parameters.

\section{Total external quantum efficiency}

Table S2 Total external quantum efficiency and thickness of the Al coating on the cathode. Since both forward and backward emission is collected, the total EQE is not expected to vary significantly with the Al thickness.

\begin{tabular}{|cc|}
\hline Al thickness/nm & EQE $\left(\cdot \mathbf{1 0}^{-3} \boldsymbol{\%}\right)$ \\
\hline 6 & 4.2 \\
10 & 3.9 \\
80 & 4.1 \\
\hline
\end{tabular}




\section{WILEY-VCH}

\section{Active layer film Morphology}

a

Figure S10
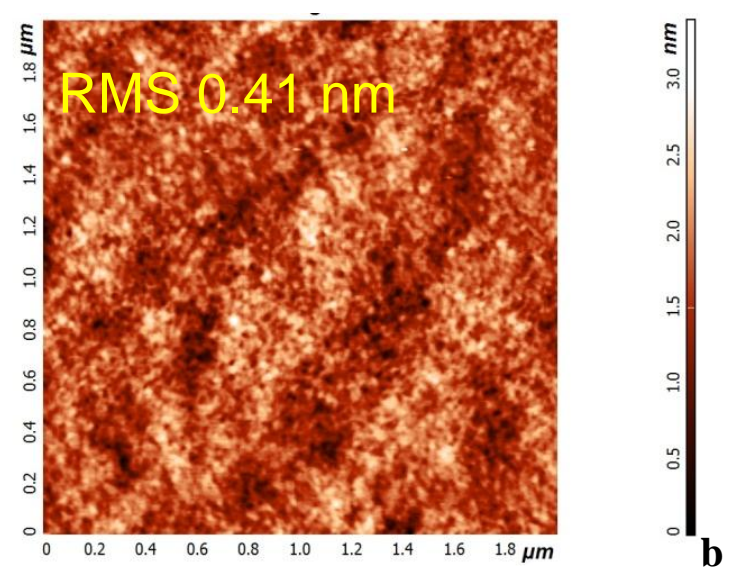
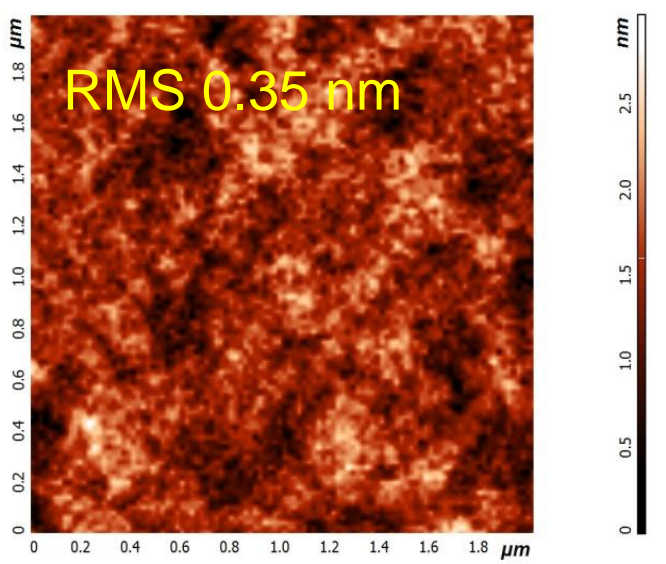

roughness (RMS) values are reported.

\section{References}

[S1] D. Shirotani, T. Suzuki, K. Yamanari, S. Kaizaki, J. Alloys Compd. 2008, 451, 325.

[S2] J. L. Lunkley, D. Shirotani, K. Yamanari, S. Kaizaki, G. Muller, Inorg. Chem. 2011, 50, 12724.

[S3] G. Hass, J. E. Waylonis, J. Opt. Soc. Am. 1961, 51, 719. 